\title{
Joint Opportunistic Scheduling of Cellular and Device-to-Device Communications
}

\author{
Rodrigo L. Batista, Carlos F. M. e Silva, \\ Tarcisio F. Maciel, José Mairton B. da Silva Jr., and Francisco R. P. Cavalcanti
}

\begin{abstract}
The joint scheduling of cellular and D2D communications to share the same radio resource is a complex task. In one hand, D2D links provide very high throughputs. In the other hand, the intra-cell interference they cause impacts on the performance of cellular communications. Therefore, designing algorithms and mechanisms that allow an efficient reuse of resources by the D2D links with a reduced impact on cellular communications is a key problem. In general, traditional Radio Resource Management (RRM) schemes (D2D grouping and mode selection) focus on finding the most compatible D2D pair for an already scheduled cellular User Equipment (UE). However, such approach limits the number of possible combinations to form the group (composed by a cellular UE and a D2D pair) to be scheduled in the radio resource. To overcome that, in this work a unified Joint Opportunistic Scheduling (JOS) of cellular and D2D communications, which is able to improve the total system throughput by exploiting the spatial compatibility among cellular and D2D UEs, is proposed. But more complexity is brought to the scheduling problem. Thus, a low-complexity suboptimal heuristic Joint Opportunistic Assignment and Scheduling (JOAS) is also elaborated. Results show that it is possible to reduce the computational complexity but still improve the overall performance in terms of cellular fairness and total system throughput with less impact on cellular communications.
\end{abstract}

Index Terms-LTE, network-assisted device-to-device communications, D2D, radio resource management, RRM, proportional fair, grouping, mode selection, joint opportunistic scheduling.

\section{INTRODUCTION}

\section{A. Background: Dealing with Additional Interference}

B Y enabling direct and low-power communication among users, hereafter generally referred as User Equipments (UEs), Device-to-Device (D2D) communication leads to an improved spectrum utilization, system capacity and/or Quality of Service (QoS) levels; and at cell boundaries, D2D links may be used as relays to extend the coverage area [1], [2].

Due to their aforementioned advantages and deployment flexibility, D2D communications are considered in 3rd Generation Partnership Project (3GPP) to facilitate Machine-Type Communication (MTC)/proximity aware services, and security/public safety applications, becoming part of Long Term Evolution (LTE) standards [3]. In this context, conventional cellular and D2D communications can be respectively referred as primary and secondary communications.

The authors are with Wireless Telecom Research Group (GTEL) at Federal University of Ceará (UFC), Fortaleza, Ceará, Brazil. E-mails: \{rodrigobatista, cfms, maciel, mairton, rodrigo\}@gtel.ufc.br.

The Associate Editor coordinating the review of this manuscript and approving it for publication was Prof. José Cândido Santos Filho.

https://doi.org/10.14209/jcis.2017.7
But, transmissions conveyed to cellular and D2D UEs on the same radio resource are coupled by intra-cell interference.

Therefore, designing algorithms and mechanisms that allow an efficient reuse of resources by the D2D links as a means to improve the spectrum utilization with a reduced impact on cellular communications is a key problem. Fig. 1 exemplifies the problem of spectrum sharing among cellular and D2D communications within a single cell.

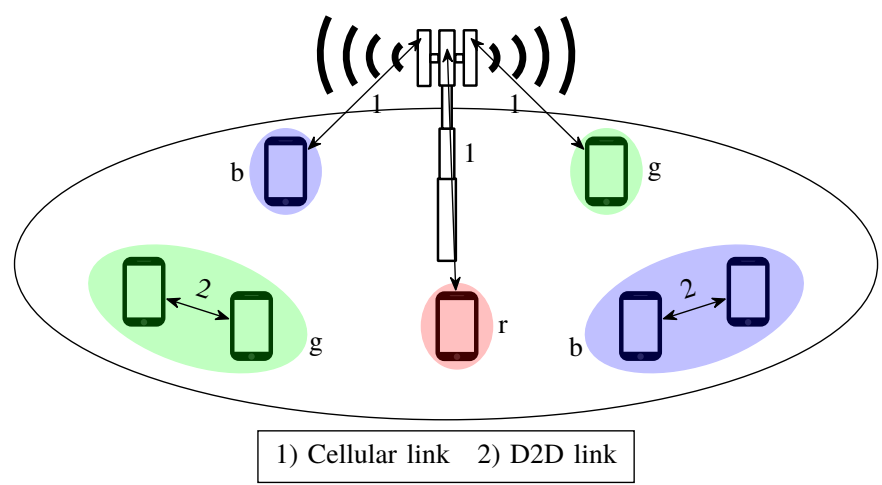

Fig. 1. An example of cellular spectrum sharing with D2D communications within a cell in downlink. The red (r) cellular UE is not in a good spatial condition for sharing resources with blue (b) and green (g) D2D UEs because it may perceive strong intra-cell interference, but blue and green cellular UEs may potentially share resources with the D2D pairs of the same color.

Several distance-based studies in the literature have shown that the efficient sharing of resources heavily depends on the distance between UEs with D2D traffic and their positions with respect to cellular UEs [1], [4]-[6]. In [4], an analysis demonstrates the feasibility in the coexistence of both communication modes (i.e., cellular and D2D communication modes, which latter are referred as mode 1 and mode 2, respectively) and shows that D2D communications bring benefits in interference-limited local area scenarios. Cellular communications happening close to Evolved Node B (eNB) (in LTE systems) and D2D communications occurring near the cell-edge provide the most favorable scenario for sharing resources. Thus, the potential benefits of D2D communications are strongly constrained by the network topology ${ }^{1}$.

The best overall capacity depends mainly on the position of the D2D transmitter relative to the cellular UE when reusing downlink resources, and to the eNB when reusing uplink resources [1]. It indicates that differentiating between

${ }^{1}$ In this work, the use of terms like spatial conditions, spatial reuse, or spatial compatibility, which are usually employed in multi-antenna systems, are related with geographical distribution of UEs and their channel conditions 
transmitters and receivers, and exploiting the UEs geographical distribution are extremely important for interference mitigation by Radio Resource Management (RRM) schemes.

\section{B. Background: Traditional RRM Schemes}

While some previous works in literature have pointed out that the overall capacity of a cellular network with underlayed D2D communications always outperforms the conventional cellular network, when cellular radio resources are reused by D2D communications in favorable conditions [1], [4], other works have proposed solutions to extend the range of situations in which D2D links are useful through RRM schemes: D2D grouping, mode selection, and power allocation [5], [7], [8].

In [5], a heuristic approach aiming at power minimization achieves a suboptimal performance in terms of spectral efficiency and throughput fairness through a joint mode selection, D2D grouping, and power allocation. Thus, RRM schemes that efficiently apply interference coordination become a major issue in cellular networks with D2D communications.

Also, most of the proposed schemes for resource assignment of D2D communications have considered a pre-selected cellular UE [1], [4], [6], [7], such that the cellular scheduling runs independently of the establishment of D2D links. In [8], the available channels are firstly allocated to cellular UEs while D2D pairs form a priority queue for each of those channels. Then, the eNB sequentially selects the D2D pair with the highest priority and sets the transmit power for each resource. In general, greedy RRM schemes solve three separate subproblems, as shown in Fig. 2.

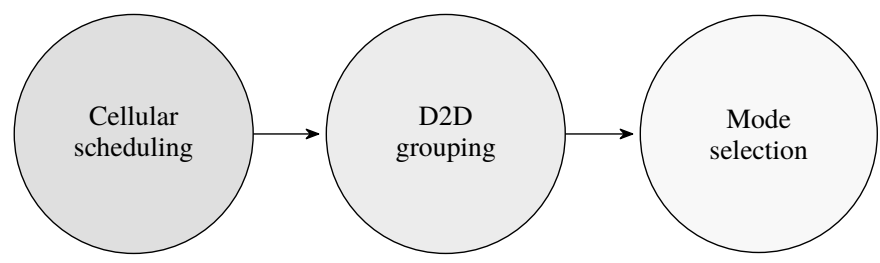

Fig. 2. RRM schemes based on a greedy approach. The cellular scheduling selects a primary cellular UE. After that, the D2D grouping step assigns a pair of D2D UEs for the primary cellular UE. Then, the mode selection is responsible for the decision-making between cellular or D2D mode [9].

However, when the primary cellular UE is chosen by a greedy approach, it is not possible to ensure that the group including this UE and a secondary D2D pair is the most spatially compatible one for sharing the radio resource. The choice of a former cellular UE that is done by these RRM schemes to be the head of a greedy search limits the exploitation of the overall multi-UE diversity. This issue is further aggravated when mode selection avoids the shared mode because the most compatible D2D pair of the primary selected cellular UE does not contribute to an increased throughput. Perhaps all available D2D pairs are spatially incompatible with that primary cellular UE, i.e., they do not have good channel conditions to share the same resource, while other cellular UEs could be prioritized instead to improve the overall spectrum utilization.

While it is known that the system capacity can be improved by exploiting the multi-UE diversity [5], the aforementioned works [1], [4], [6]-[8] have neglected the benefits of such diversity, as they just focused on finding the most spatially compatible D2D pairs with respect to a scheduled cellular UE.

Besides that, since the instantaneous throughput information of all cellular UEs and D2D pairs must already be available within a cell for mode selection purposes, these measurements may be used instead in a unified framework for joint resource assignment of cellular and D2D communications.

Moreover, in [10] the authors claim that they consider a joint mode selection, channel assignment, and power control to maximize the overall system throughput. Three modes are then considered: dedicated cellular mode (herein mode 1), dedicated D2D mode, and reuse mode (herein mode 2).

However, the optimization problem is decomposed into two subproblems: transmit power control for both cellular and D2D UEs, and joint mode selection and channel assignment for each D2D UE. Thus, and once again, a greedy approach is followed.

\section{Problem Statement}

To the best of our knowledge, prior works have not proposed an optimization model for resource assignment that takes into account cellular scheduling, D2D grouping, and mode selection in a unified framework aiming to exploit the multiUE diversity and to provide fairness. Hence, we propose the unified Joint Opportunistic Scheduling (JOS) problem for resource assignment among cellular and D2D communications, as shown in Fig. 3. The JOS problem allows opportunistic exploitation of instantaneous channel fluctuations and prioritizes communications which are in better conditions for sharing resources as to obtain an improved system capacity.

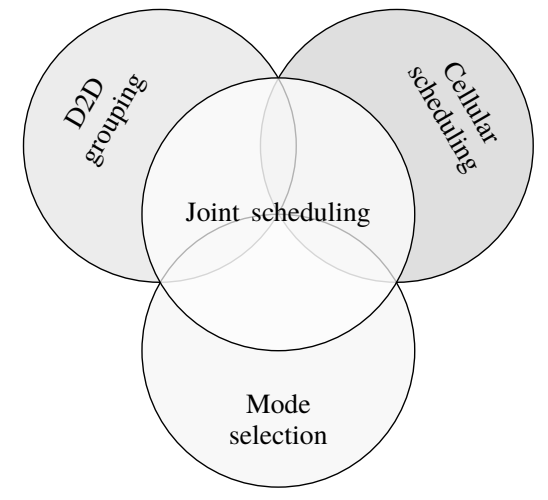

Fig. 3. Proposed JOS problem for joint scheduling of cellular and D2D communications.

To avoid an Exhaustive Search (ES) over all possible resource assignments, scheduling, and mode selection decisions, simple and effective heuristic algorithms are required for alleviating concerns about processing complexity. Hence, we propose the Joint Opportunistic Assignment and Scheduling (JOAS) heuristic as an efficient low-complexity solution for the JOS problem. The proposed heuristic forms various candidate groups and evaluates them using a modified Proportional Fair (PF) metric, but processing only the UEs and groups most likely to be scheduled.

In addition, assuming that the maximum throughput in mode 2 is achieved when only one D2D pair is enabled per 
cell at each scheduling instance in a LTE network [9], [10], especially in small cell cases, the JOAS heuristic limits the number of possible combinations to group only one cellular $\mathrm{UE}$ and one D2D pair.

Besides that, it also considers a mechanism to limit the impact of D2D communications on the performance of cellular communications. The main contributions of this work are summarized as follows:

1) Propose the JOS problem whose objective is to maximize a modified PF metric that takes into account the instantaneous throughput of celular and D2D communications;

2) Elaborate a low-complexity suboptimal JOAS heuristic to solve the JOS problem, which is based on resource assignment and joint scheduling of groups of cellular and D2D UEs, along with optional pre-selection schemes and a protection mechanism for cellular communications.

The remaining sections of this manuscript are organized in the following manner. Section II presents the system modeling. In Section III, a formulation for the JOS problem is proposed, while Section IV describes the JOAS heuristic. In Section V traditional RRM schemes are briefly described. Simulation results are presented and discussed in Section VI. Finally, in Section VII, conclusions and future perspectives are drawn.

\section{System Modeling}

In this section the system model is addressed. Let us assume a multi-cell LTE network with $C$ cells. The shape of each cell is a regular hexagon with an eNB placed in its center [11].

To model a scenario where D2D communications are likely to happen underlaying such network, a percentage of the total $J$ UEs within the cell coverage area is clustered inside a rectangular hotspot zone located near the cell-edge (which ensures the favorable conditions for sharing radio resources [6]), while the others are uniformly distributed over the remaining area [12]. As an example, for a percentage of $25 \%$, if there are $J=16$ UEs per cell, $J_{\mathrm{CELL}}=12$ of them are cellular UEs and $J_{\mathrm{D} 2 \mathrm{D}}=4$ are D2D UEs.

Considering that UEs inside the hotspot are very close to each other, and far away from most cellular UEs, D2D pairs are obtained by randomly pairing those UEs (transmitter and receiver). Following the previous example, with $J_{\mathrm{D} 2 \mathrm{D}}=4 \mathrm{D} 2 \mathrm{D}$ UEs there exist $M=2$ D2D pairs per cell.

LTE systems employ Orthogonal Frequency Division Multiple Access (OFDMA) for multi-UE transmissions in downlink communication direction. As such, subcarriers are grouped in blocks of 12 adjacent subcarriers spaced by $15 \mathrm{kHz}$, which gives a total bandwidth of $180 \mathrm{kHz}$ per block. The information is transported on that bandwidth over a slot that lasts for $0.5 \mathrm{~ms}$ and seven Orthogonal Frequency Division Multiplexing (OFDM) symbols. This frequency-time block is designated as Physical Resource Block (PRB) and it is the minimum allocable radio resource unit. Nevertheless, and due to practical reasons, each scheduled UE takes two slots (a subframe).

To this end, the scheduling happens at each Transmission Time Interval (TTI) (1 ms) on a PRB basis for the total number of $N$ PRBs, that are fully reused in all cells (reuse-1).

Moreover, the channel response for each PRB is represented by the complex channel coefficient associated with its middle subcarrier and first OFDM symbol; and the channel coherence bandwidth is assumed to be larger than the bandwidth of a single PRB, leading to a flat fading channel over each of them. Furthermore, modeling the complex channel coefficients includes the propagation effects on wireless channels, namely, pathloss, shadowing, and fast fading for the urban-microcell environment [11], [13], [14].

Now, let us assume that $t(m)$ and $r(m)$ represent, respectively, the transmitter and receiver that belong to the D2D pair $m \in\{0,1, \ldots, M\}$, and that $j \in\left\{1,2, \ldots, J_{\text {CELL }}\right\}$ denotes a cellular UE. Note that $m=0$ represents the case where there are only cellular UEs. Also, in the multi-cell network, eNBs (likewise cells ${ }^{2}$ ) are referred by their index $c \in\{1,2, \ldots, C\}$.

The total transmit power, $P_{\mathrm{UE}}$ from a UE and $P_{\mathrm{CELL}}$ from an eNB, is divided among the allocated PRBs. Hence, the transmit power per resource $n \in\{1,2, \ldots, N\}$ for the cellular link is denoted as $p_{c, n}$ and for the D2D link as $p_{t(m), c, n}$; and the cellular link's channel is represented by $h_{j, c, n} \in \mathbb{C}$, while $h_{r(m), t(m), c, n} \in \mathbb{C}$ translates the channel of D2D pair $m$ within cell $c$ at PRB $n$. And, the reported Channel State Information (CSI) from all involved UEs does not suffer any error or delay, i.e., the CSI knowledge is perfect.

Admitting $\eta^{2}$ as the average power of additive white Gaussian noise at receivers in downlink, the Signal-to-Interferenceplus-Noise Ratios (SINRs) $\gamma_{j, m, c, n}^{\mathrm{CELL}}$ and $\gamma_{j, m, c, n}^{\mathrm{D} 2 \mathrm{D}}$ perceived, respectively, by the cellular UE $j$ and the D2D receiver $r(m)$ may be written as presented in (1). Table I has the description of channels and transmit powers and Fig. 4 illustrates them for a better understanding.

TABLE I

DESCRIPTION OF CHANNELS AND TRANSMIT POWERS IN (1)

\begin{tabular}{|c|c|}
\hline Varia & Description \\
\hline $\begin{array}{l}h_{j, c, n} \\
h_{j, t(m), c, n}\end{array}$ & $\begin{array}{l}\text { Desired channel between eNB } c \text { and } \mathrm{UE} j \text { at } \mathrm{PRB} n \\
\text { Interfering channel between D2D transmitter } t(m) \\
\text { within cell } c \text { and UE } j \text { at PRB } n\end{array}$ \\
\hline $\begin{array}{l}h_{j, c^{\prime}, n} \\
h_{j, t}\left(m^{\prime}\right), c^{\prime}, n\end{array}$ & $\begin{array}{l}\text { Interfering channel between eNB } c^{\prime} \text { and UE } j \text { at PRB } n \\
\text { Interfering channel between D2D transmitter } t\left(m^{\prime}\right) \\
\text { within cell } c^{\prime} \text { and UE } j \text { at PRB } n\end{array}$ \\
\hline$h_{r(n}$ & $\begin{array}{l}\text { Desired channel between D2D transmitter } t(m) \text { and } \\
\text { receiver } r(m) \text { within cell } c \text { at PRB } n\end{array}$ \\
\hline$h_{r(m), c, n}$ & $\begin{array}{l}\text { Interfering channel between eNB } c \text { and receiver } r(m) \\
\text { at PRB } n\end{array}$ \\
\hline$h_{r(m), c^{\prime}, n}$ & $\begin{array}{l}\text { Interfering channel between eNB } c^{\prime} \text { and receiver } r(m) \\
\text { at PRB } n\end{array}$ \\
\hline$h_{r(m}$ & $\begin{array}{l}\text { Interfering channel between D2D transmitter } t\left(m^{\prime}\right) \\
\text { within cell } c^{\prime} \text { and receiver } r(m) \text { at PRB } n\end{array}$ \\
\hline$p_{c, n}$ & Transmit power from eNB $c$ at PRB $n$ \\
\hline$p_{t(m), c, n}$ & $\begin{array}{l}\text { Transmit power from D2D transmitter } t(m) \text { within } \\
\text { cell } c \text { at PRB } n\end{array}$ \\
\hline & Transmit power from eNB $c^{\prime}$ at PRB $n$ \\
\hline$p_{t\left(m^{\prime}\right)}$ & $\begin{array}{l}\text { Transmit power from D2D transmitter } t\left(m^{\prime}\right) \text { within } \\
\text { cell } c^{\prime} \text { at PRB } n\end{array}$ \\
\hline
\end{tabular}

For this work we restrict ourselves to the downlink communication direction, no power control is used, and a single omnidirectional antenna is installed in all eNBs and UEs. In the case of multiple antenna systems, or the uplink direction, or

${ }^{2}$ In this work, the terms eNB and cell are sometimes used interchangeably 


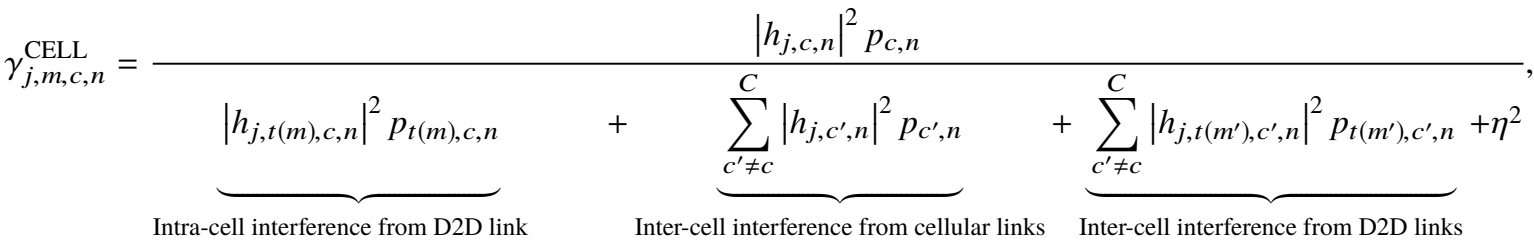

$$
\begin{aligned}
& \gamma_{j, m, c, n}^{\mathrm{D} 2 \mathrm{D}}=\frac{\mid \underbrace{\left|h_{r(m), t(m), c, n}\right|^{2} p_{t(m), c, n}}_{\text {Intra-cell interference from cellular link }}+\underbrace{\sum_{c^{\prime} \neq c}^{C}\left|h_{r(m), c^{\prime}, n}\right|^{2} p_{c^{\prime}, n}}_{\text {Inter-cell interference from cellular links }}+\underbrace{\sum_{c^{\prime} \neq c}^{\sum_{c}}\left|h_{r(m), t\left(m^{\prime}\right), c^{\prime}, n}\right|^{2} p_{t\left(m^{\prime}\right), c^{\prime}, n}+\eta^{2}}_{\text {Inter-cell interference from D2D links }}}{.}
\end{aligned}
$$

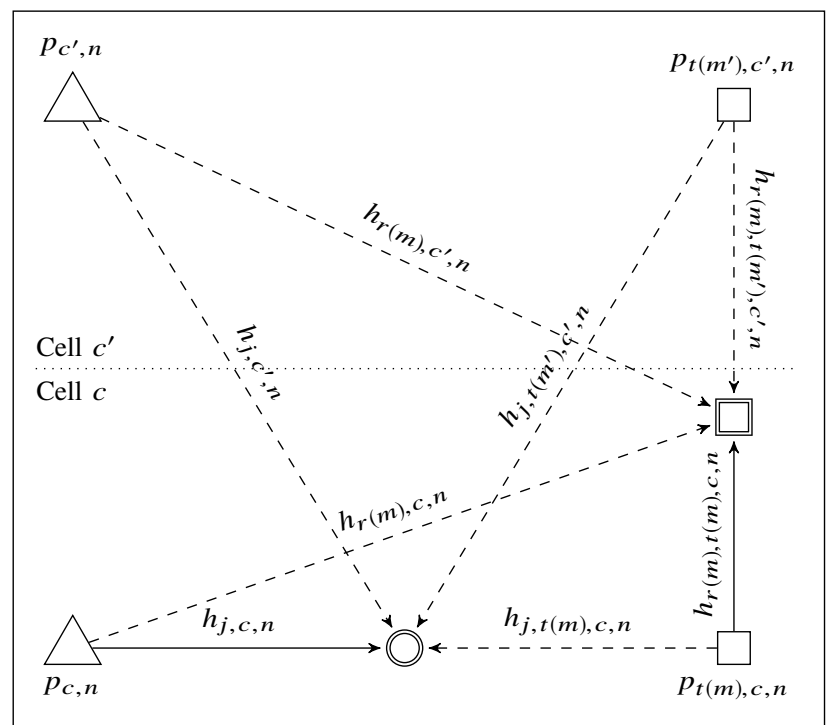

Fig. 4. Illustration of channels and transmit powers in (1); where triangle represents an eNB, circle is a cellular UE, and square is a D2D UE. The double line (circle and square) indicates a receiver. Moreover, solid lines are for desired links while dashed lines represent interfering links.

allowing that multiple D2D pairs to be grouped with a cellular $\mathrm{UE}$, the required modifications in (1) shall be straightforward.

In a given TTI $t$ (note that, whenever possible, and to simplify notation, the index $t$ is omitted from the following equations) and considering that the link adaptation function $f_{\mathrm{LA}}(\cdot)$ selects the Modulation and Coding Scheme (MCS) which yields the maximum data rate [15], [16], the throughput of cellular and D2D links is calculated as follows

$$
\begin{aligned}
& R_{j, m, c, n}^{\mathrm{CELL}}=f_{\mathrm{LA}}\left(\gamma_{j, m, c, n}^{\mathrm{CELL}}\right), \\
& R_{j, m, c, n}^{\mathrm{D} 2 \mathrm{D}}=f_{\mathrm{LA}}\left(\gamma_{j, m, c, n}^{\mathrm{D} 2 \mathrm{D}}\right) .
\end{aligned}
$$

In the cellular scheduling without D2D communication, i.e., $m=0$, a standard PF metric $\psi_{j, 0, c, n}^{\mathrm{STD}}$ is chosen. Such scheduling is performed by estimating the instantaneous throughput $R_{j, 0, c, n}^{\mathrm{CELL}}$ in each resource $n$ and updating the average throughput $T_{j, 0, c}^{\mathrm{CELL}}$ [17]; the low-pass filtered average throughput of UE $j$ after transmission at TTI $t+1$ is calculated in the following manner

$$
T_{j, 0, c}^{\mathrm{CELL}}(t+1)=\left(1-\frac{1}{\Delta_{\mathrm{PF}}}\right) T_{j, 0, c}^{\mathrm{CELL}}(t)+\frac{1}{\Delta_{\mathrm{PF}}} \sum_{n}^{N} R_{j, 0, c, n}^{\mathrm{CELL}}(t),
$$

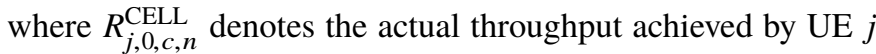
within cell $c$ at PRB $n$ and TTI $t$, and $\Delta_{\mathrm{PF}}$ is the length of the exponentially weighted time window. Thus, resources are sequentially allocated to each UE $j^{\star}$ with the best PF metric, particularly

$$
j^{\star}=\left\{\begin{aligned}
\underset{j}{\arg \max }\left\{\psi_{j, 0, c, n}^{\mathrm{STD}}\right\}, & \text { if } R_{j, 0, c, n}^{\mathrm{CELL}} \geq R_{\min }, \\
\emptyset, & \text { otherwise }
\end{aligned}\right.
$$

where

$$
\psi_{j, 0, c, n}^{\mathrm{STD}}=\frac{R_{j, 0, c, n}^{\mathrm{CELL}}}{T_{j, 0, c}^{\mathrm{CELL}}}
$$

and $R_{\text {min }}>0$ denotes the instantaneous throughput provided by the lowest MCS per PRB in LTE systems.

\section{Joint Opportunistic Scheduling Problem}

The objective of the current section is to propose a unified optimization problem for the Joint Opportunistic Scheduling (JOS) of cellular and D2D communications. The JOS problem is designed so that it persecutes the maximization of the $\mathrm{PF}$ ratio and is based on the same priority calculation performed by the PF metric in (4). However, the instantaneous cellular throughput is modified to the total instantaneous throughput $R_{j, m, c, n}^{\mathrm{TOTAL}}$, which is given as

$$
R_{j, m, c, n}^{\mathrm{TOTAL}}=R_{j, m, c, n}^{\mathrm{CELL}}+R_{j, m, c, n}^{\mathrm{D} 2 \mathrm{D}},
$$

where $R_{j, m, c, n}^{\mathrm{CELL}}$ and $R_{j, m, c, n}^{\mathrm{D} 2 \mathrm{D}}$ are, respectively, the instantaneous throughput values of the group $(j, m)_{c, n}$ (hereafter, indexes $c$ and $n$ are omitted for notation simplicity) composed by the cellular UE $j$ and D2D pair $m$ within cell $c$ while sharing the same PRB $n$. Hence, the modified priority $\psi_{j, m, c, n}^{\mathrm{JOS}}$ of each group is calculated as follows

$$
\psi_{j, m, c, n}^{\mathrm{JOS}}=\frac{R_{j, m, c, n}^{\mathrm{TOTAL}}}{T_{j, 0, c}^{\mathrm{CELL}}} .
$$


The principle behind the standard PF in (4) is to schedule the UE $j^{\star}$ which has the largest ratio between the achievable instantaneous throughput and the average one. Looking to (6) the same principle applies as before, but for the group $(j, m)$. Therefore, if for any reason the celular UE experiences poor channel conditions but the D2D pair may achieve large throughput, the group $\left(j^{\star}, m^{\star}\right)$ is scheduled. In addition, when a cellular UE $j$ is not scheduled for several TTIs, its average throughput $T_{j, 0, c}^{\text {CELL }}$ is low, which further larges (6). As such, the fairness among cellular UEs is still preserved.

Defining an optimization binary variable $x_{j, m, c, n}$, the JOS problem can be formulated as

$$
x_{j, m, c, n}^{\star}=\underset{x_{j, m, c, n}}{\arg \max }\left\{\sum_{j}^{J_{\mathrm{CELL}}} \sum_{m}^{M} \sum_{c}^{C} \sum_{n}^{N} x_{j, m, c, n} \psi_{j, m, c, n}^{\mathrm{JOS}}\right\},
$$

subject to

$$
\begin{aligned}
& \sum_{j}^{J_{\mathrm{CELL}}} \sum_{m}^{M} x_{j, m, c, n} \leq 1, \forall c, n, \\
& x_{j, m, c, n} \in\{0,1\}, \forall j, m, c, n, \\
& R_{j, m, c, n}^{\mathrm{CELL}} \geq x_{j, m, c, n} R_{\text {min }}, \forall j, m, c, n, \\
& R_{j, m, c, n}^{\mathrm{D} 2 \mathrm{D}} \geq x_{j, m, c, n} R_{\text {min }}, \forall j, c, n \text { and } m>0,
\end{aligned}
$$

where $R_{\text {min }}$ denotes the instantaneous throughput provided by the lowest MCS per PRB in LTE systems.

On the one hand, looking to (7a) and from (6), (5), (2), and (1) it can be easily observed that $\psi_{j, m, c, n}^{\mathrm{JOS}}$ is intrinsically related with the transmit power, which is the coupling element between cellular scheduling, D2D grouping, and mode selection subproblems. Therefore, if $x_{j, m, c, n}$ equals zero, i.e., the group $(j, m)$ is not scheduled to transmit, then the allocated power to channels $h_{j, c, n}$ and $h_{r(m), t(m), c, n}$ in (1) is also zero.

On the other hand, the instantaneous throughput estimated by the scheduling policy needs to accurately reflect the achievable throughput in each radio resource [17]. However, such information is known only after power allocation is performed, which may be unavailable for the scheduling policy [18]. To simplify this problem, Equal Power Allocation (EPA) is used.

The full reuse of PRBs combined with EPA in the downlink direction permits the exact knowledge of the transmit power by the scheduling policy at eNB (i.e., $p_{c, n}=p_{c^{\prime}, n}=P_{\mathrm{CELL}} / N$ ). However, for D2D UEs, the number of resources is allocated opportunistically and, therefore, the transmit power (i.e., $p_{t(m), c, n}$ and $\left.p_{t\left(m^{\prime}\right), c^{\prime}, n}\right)$ is hard to be predicted. In such case, a realistic prediction of the average transmit power per resource for each UE at TTI $t+1$ is computed based on an exponential moving average as in [18]

$$
\begin{aligned}
& \hat{p}_{t(m), c, n}(t+1)= \\
& \left\{\begin{aligned}
\left(1-\frac{1}{\Delta_{\mathrm{AVG}}}\right) \hat{p}_{t(m), c, n}(t)+\frac{1}{\Delta_{\mathrm{AVG}}} p_{t(m), c, n}(t), & \text { if } m=m^{\star}, \\
\hat{p}_{t(m), c, n}(t), & \text { otherwise, }
\end{aligned}\right.
\end{aligned}
$$

where $P_{\mathrm{UE}} / N \leq \hat{p}_{t(m), c, n} \leq P_{\mathrm{UE}}$ is the estimated average transmit power from D2D transmitter $t(m)$ within cell $c$ at PRB $n$ used by the scheduling policy, $p_{t(m), c, n}$ is the transmit power effectively allocated, and $\Delta_{\mathrm{AVG}}$ is the length in TTIs of the exponentially weighted time window.

It is clear that (7) belongs to the family of Mixed-integer Nonlinear Programming (MINLP) problems, which combine the combinatorial difficulty of optimizing over discrete variable sets with the challenges of handling nonlinear functions [19], and therefore is a Nondeterministic Polynomial Time (NP)-hard combinatorial problem, which is intractable.

\section{Joint Opportunistic Assignment and Scheduling Heuristic}

In this section, a heuristic called JOAS is elaborated as a low-complexity solution for the JOS problem stated in (7). JOAS runs within each cell on a PRB basis independently from other cells. In this manner, for notation simplicity, indexes $c$ and $n$ are omitted from the following equations.

Based on the principle of a multi-UE scheduling, the main idea of JOAS heuristic is to improve opportunistically the total throughput of cellular and D2D communications. In order to avoid testing all combinations of cellular UEs and D2D pairs, the proposed method allows the processing of candidate groups most likely to be scheduled in each PRB. For this to happen, the heuristic is divided into two main steps (see Fig. 5):

- Using a certain metric to measure the spatial compatibility among cellular UEs and D2D pairs, assignment selects the most spatially compatible D2D pair to each cellular UE. Thus, it enables the processing for scheduling of only a reduced set among all possible candidate groups that would be considered by an ES;

- The scheduling that occurs jointly and opportunistically prioritizes groups that were assigned by the previous step whenever it is possible to increase the total throughput. Hence, an implicit mode selection happens, i.e., either the scheduling of a group $\left(j^{\star}, m^{\star}\right)$, hereafter referred as mode 2 , or the scheduling of just a cellular UE $j^{\star}$, without resource sharing, denoted as mode 1 , when the UE alone achieves a largest throughput.

Furthermore, some optional mechanisms are also designed to reduce the processing complexity by pre-selecting UEs and protect cellular communications:

- The pre-selection schemes, namely pre-assignment and pre-scheduling schemes, avoid a full wide-search on the unlikely scheduling candidates [20]. They reduce processing complexity of both assignment and scheduling steps by selecting only the most likely cellular UEs and groups to be scheduled;

- The protection mechanism avoids the selection of D2D pairs that would highly harm the performance of cellular communications, by focusing on the total throughput and interference coordination.

\section{A. Assignment}

As said before, the assignment step is designed to avoid the increasing complexity in terms of throughput calculations for the scheduling step. So, the assignment selects the most likely D2D pair to be scheduled with each cellular UE. Herein, an assignment metric $\phi_{j, m}$ that measures the spatial compatibility 


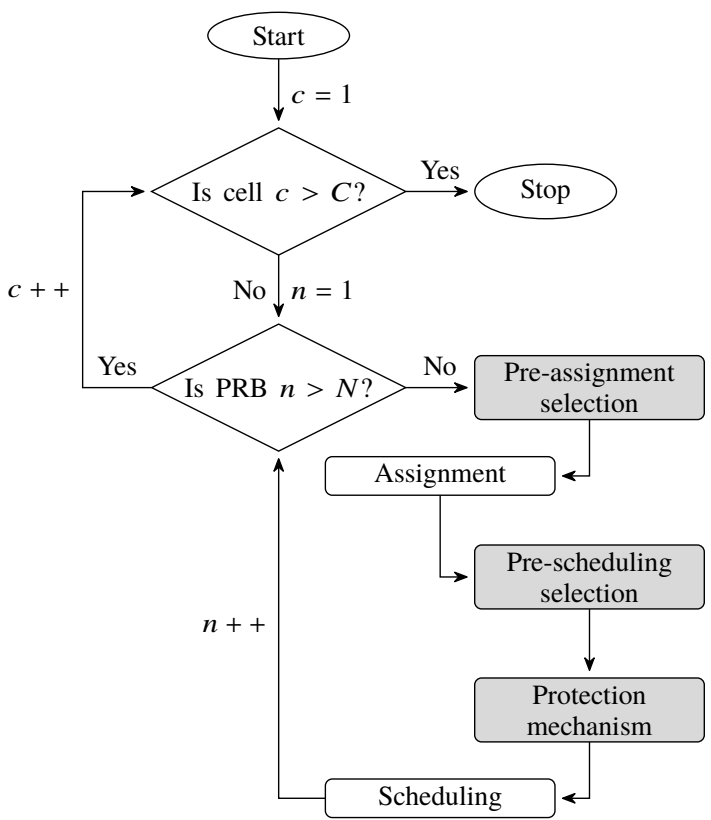

Fig. 5. Flowchart of JOAS heuristic; gray blocks indicate they are optional.

of each cellular UE $j$ and D2D pair $m$ is employed. Therefore, the most compatible D2D pair for the cellular UE $j$ is assigned as follows

$$
\left(j, m^{\star}\right)=\underset{m}{\arg \max }\left\{\phi_{j, m}\right\} .
$$

Different link measurements can be used for the assignment metric. These measurements try to capture the effects related with spectral efficiency gain and interference reduction. Also, depending on the measurement, the assignment may be done in a slow time basis (e.g., seconds) and at once for all PRBs (i.e., whole bandwidth). To avoid increasing the computational complexity and overhead on reporting measurements to the eNB, the assignment metric shall be as simple as possible.

As the desired and interfering cellular links are assumed to be known at the eNB, and the D2D link is presumed to be known by the communicating UEs, only the D2D interfering link at the cellular UE is difficult to obtain. In this case, e.g., a minimum reference power used by the D2D discovery procedure over that link [21] can be used to infer the channel $h_{j, t(m), c, n}$ in Fig. 4.

The assignment metrics are detailed in the following and the measurements required by each of them are indicated in Table II, whereas channels are described in Table I (apart from cell and PRB indexes), and $\hat{z}_{j}$ and $\hat{z}_{r(m)}$ are, respectively, the interference estimation suffered by the cellular UE $j$ and D2D receiver $r(m)$.

TABLE II

LINK MEASUREMENTS REQUIRED BY EACH ASSIGNMENT METRIC

\begin{tabular}{lcccccc}
\hline & $h_{j}$ & $h_{r(m), t(m)}$ & $h_{r(m)}$ & $h_{j, t(m)}$ & $\hat{z}_{j}$ & $\hat{z}_{r(m)}$ \\
\hline RND & $x$ & $x$ & $x$ & $x$ & $x$ & $x$ \\
SLNR & $x$ & $\checkmark$ & $x$ & $\checkmark$ & $x$ & $x$ \\
ES & $\checkmark$ & $\checkmark$ & $\checkmark$ & $\checkmark$ & $\checkmark$ & $\checkmark$ \\
\hline
\end{tabular}

1) Random (RND): The RND metric randomly selects a $\mathrm{D} 2 \mathrm{D}$ pair such that each pair within the cell has equal chances to be scheduled [6]. It configures a scenario with non-networkassisted D2D communications, that does not take into account any link measurement, and, therefore, disfavors the spatial compatibility among cellular UEs and D2D pairs. This metric is employed only to obtain a lower bound on the performance of the assignment step.

2) Signal-to-Leakage-plus-Noise Ratio (SLNR): The SLNR is based on the ratio between the desired signal power over the undesired leakage of a D2D communication. Measurements of the channel gain and generated interference (leakage) are closely related to good and bad spatial conditions for sharing resources. Thus, this metric makes the differentiation among D2D transmitters (interferes) and cellular receivers to measure how good is the geographical separation between them. The SLNR metric is given by

$$
\phi_{j, m}=\frac{\left|h_{r(m), t(m)}\right|^{2} p_{t(m)}}{\left|h_{j, t(m)}\right|^{2} p_{t(m)}+\eta^{2}},
$$

where $\left|h_{r(m), t(m)}\right|^{2}$ is the channel gain between the transmitter and receiver of D2D pair $m, p_{t(m)}$ is the transmit power from D2D transmitter $t(m)$, and $\left|h_{j, t(m)}\right|^{2}$ is the interfering channel gain between D2D transmitter $t(m)$ and celular UE $j$, whereby $\left|h_{j, t(m)}\right|^{2} p_{t(m)}$ is the leakage.

3) Exhaustive Search (ES): The ES metric ${ }^{3}$ estimates the achievable throughput per group of all possible $(j, m)$ groups, and assigns to the cellular UE $j$ the D2D pair $m$ that leads to the highest modified PF priority, expressed in (6). The ES metric is given by

$$
\phi_{j, m}=\psi_{j, m}^{\mathrm{JOS}} .
$$

The time complexity (number of comparisons) in both (11) and (10) is expected to be very similar. However, the computational complexity for ES metric shall be higher, especially for situations of high UE density, since it depends on (6), (5), (2), and (1). This solution is not necessarily optimal and it is only used as upper bound on the performance of assignment step.

\section{B. Scheduling}

The scheduling step makes a joint opportunistic processing of the estimated instantaneous throughput values of both cellular and D2D links to get improved total gains. Each radio resource is mandatorily allocated to one cellular UE while its sharing with a D2D pair depends on the total throughput. This step has the flexibility of avoiding the schedule of a D2D communication when it does not provide additional gain to the total throughput, thus performing mode selection. The scheduling is as follows

$$
\left(j^{\star}, m^{\star}\right)=\underset{j}{\arg \max }\left\{\max \left\{\psi_{j, 0}^{\mathrm{STD}}, \psi_{j, m^{\star}}^{\mathrm{JOS}}\right\}\right\},
$$

where $m^{\star}=0$ represents the mode 1 case, which schedules the cellular UE $j^{\star}$ through the standard PF metric (4b); and $m^{\star} \neq$

\footnotetext{
${ }^{3}$ Strictly speaking, ES should not be considered as a metric because it just searches over the space of all possible solutions and selects the best one.
} 
0 represents the mode 2 case, which schedules the cellular $\mathrm{UE} j^{\star}$ and D2D pair $m^{\star}$ through the JOS metric (6).

Furthermore, some additional comments on scheduling are worth to be highlighted:

- Groups compete for resources by using the JOS metric, which has its historical average throughput values based only on the cellular throughput. So, as explained in Section III, the fairness obtained by (12) is still guaranteed for cellular UEs such as by the standard PF in (4);

- Cellular UEs in groups with low spatial compatibility tend to be scheduled in mode 1 because of the low total throughput in mode 2;

- In mode 2, cellular UEs always have their instantaneous throughput reduced due to the intra-cell interference from D2D link. However, in favorable conditions for resource sharing, i.e., with a large total instantaneous throughput, they still have high priority to be selected in subsequent scheduling rounds because of the slow increase on historical throughput compared to mode 1 .

\section{Pre-selection Schemes}

The pre-selection schemes alleviate concerns with the extensive processing for scenarios with high UE density through a controlled trade-off between capacity and complexity [20]. Herein, pre-selection schemes are applied to reduce the complexity of assignment and scheduling steps. While the preassignment scheme allows the assignment processing only for those cellular UEs most likely to be scheduled, the prescheduling scheme allows the scheduling processing only for the groups most likely to be scheduled. In the following, both pre-selection schemes are detailed.

1) Pre-assignment: It reduces the complexity of the assignment step, and consequently of the scheduling step, by picking up only a fraction $F_{A}$ of those UEs most likely to be scheduled in mode 1. Therefore, the assignment step is dealt only to a subset $\mathcal{A} \subset \mathcal{J}_{\text {CELL }}$ of the total set $\mathcal{J}_{\text {CELL }}$ of cellular UEs within the cell, with $|\mathcal{A}|=F_{A}\left|\mathcal{J}_{\text {CELL }}\right|$, where $|\cdot|$ is the cardinality operator (recall that $\left|\mathcal{J}_{\text {CELL }}\right|=J_{\text {CELL }}$ ) and $F_{A}$ is a fraction of cellular UEs with the highest standard PF priority values.

2) Pre-scheduling: The complexity of the scheduling step is reduced by allowing total throughput calculations only for a fraction $F_{S}$ of the most spatially compatible groups, which are likely to be scheduled in mode 2 . From the groups $\mathcal{G}$ provided by the assignment step, total throughput calculations are dealt only to a subset $\mathcal{S} \subset \mathcal{G}$ of groups, with $|\mathcal{S}|=F_{S}|\mathcal{G}|$, where $F_{S}$ is a fraction of groups with the highest assignment metric values.

\section{Protection Mechanism}

As the total throughput may hide a low cellular throughput because of a high D2D throughput, a protection mechanism is designed to prevent the negative impact of excessive interference from D2D link on the performance of cellular communications. It protects the cellular UEs by ensuring a minimum throughput requirement for cellular communications.

Since it is performed before the scheduling step, mode 2 can be avoided when the loss on the instantaneous throughput of a cellular UE due to the generated interference of its assigned $\mathrm{D} 2 \mathrm{D}$ pair is higher than the maximum allowable one.

Let $f_{j, m^{\star}}$ denote the percentage throughput loss of a cellular UE $j$ due to the impact of its assigned D2D pair $m^{\star}$, which is given by

$$
f_{j, m^{\star}}=1-\frac{R_{j, m^{\star}}^{\mathrm{CELL}}}{R_{j, 0}^{\mathrm{CELL}}},
$$

where $R_{j, 0}^{\mathrm{CELL}}$ is the instantaneous throughput of the cellular UE $j$ in mode 1 and $R_{j, m^{\star}}^{\mathrm{CELL}}$ in mode 2 .

As such, the protection mechanism switches off a candidate group by using a reversed step function to nullify its JOS priority (thus avoiding mode 2) as follows

$$
\psi_{j, m^{\star}}^{\mathrm{JOS}}=\left\{\begin{aligned}
\psi_{j, m^{\star}}^{\mathrm{JOS}}, & \text { if } f_{j, m^{\star}} \leq F_{I}, \\
0, & \text { otherwise, }
\end{aligned}\right.
$$

where $F_{I}$ denotes the maximum admissible throughput percentage loss of a cellular communication when D2D communications are enabled for sharing resources, compared to a baseline scenario with only cellular communications $\left(F_{I}=0 \%\right)$.

Power control methods at D2D transmitters for interference mitigation are desired to minimize the impact on cellular communications. However, such methods are out of the scope of this work, since they would mask the benefits of JOAS.

\section{Traditional Radio Resource Management Schemes}

For comparison with JOAS, in this section traditional RRM schemes used to enable the sharing of radio resources between cellular and D2D communications are explained. Usually such schemes divide the JOS problem (7) into cellular scheduling, D2D grouping, and mode selection subproblems. An algorithmic description of these procedures is provided in Fig. 6 and detailed as follows:

1) The standard PF scheduler selects the primary cellular UE $j^{\star}$ according to the priority $\psi_{j, 0}^{\mathrm{STD}}$ defined in (4);

2) The D2D grouping scheme assigns the most spatially compatible D2D pair $m^{\star}$ to the primary cellular UE $j^{\star}$ by using the assignment metric in (9);

3) The mode selection algorithm only allows D2D communications when the total throughput in mode 2 is higher than in mode 1. Moreover, the cellular throughput is also protected such that it must be higher than zero.

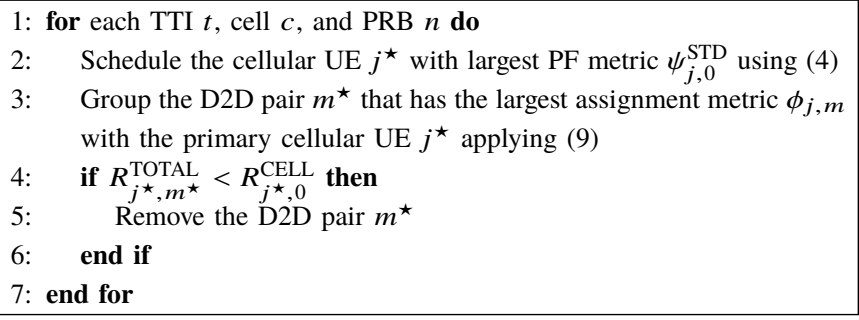

Fig. 6. RRM schemes: cellular scheduling (line 2), D2D grouping (line 3), and mode selection (lines 4 to 6).

In the following, GRP refers to D2D grouping and GRP+MS to D2D grouping with mode selection. 


\section{Results And Analysis}

This section provides a performance assessment for JOAS heuristic in the multi-cell scenario through system-level simulations aligned with LTE systems [11], [13], [14], as described in Section II. The main simulation parameters are in Table III. Note that when the number of UEs in the system increases, the dimension (complexity) of the problem also increases, scaling exponentially, which brings computational issues. Therefore, the maximum number of UEs per cell is fixed to 16 .

TABLE III

SiMULATION PARAMETERS

\begin{tabular}{|c|c|c|}
\hline Parameter & Value & Ref. \\
\hline Number of cells $(C)$ & 7 (with wrap-around) & \\
\hline Cellular environment & Urban-microcell & {$[11]$} \\
\hline Inter-site distance & $500 \mathrm{~m}$ & {$[11]$} \\
\hline eNB transmit power & $38 \mathrm{dBm}$ & [13] \\
\hline UE transmit power & $24 \mathrm{dBm}$ & [13] \\
\hline Cellular pathloss model & $34.5+38 \log _{10}(d), d$ in meter & [13] \\
\hline Shadowing std. dev. & $10 \mathrm{~dB}$ & \\
\hline Antenna configuration & SISO, omnidirectional & {$[13]$} \\
\hline Fast fading model & 3GPP SCM & {$[11]$} \\
\hline Average UE speed & $3 \mathrm{~km} / \mathrm{h}$ & {$[13]$} \\
\hline Hotspot size (width $\times$ height) & $120 \mathrm{~m} \times 50 \mathrm{~m}$ & \\
\hline UEs per cell $(J)$ & $4,8,12$, and 16 & \\
\hline Percentage of hotspot UEs & $50 \%(M=1,2,3$, and 4$)$ & \\
\hline UE distribution & Uniform & \\
\hline D2D pathloss model & $37+30 \log _{10}(d), d$ in meter & {$[14]$} \\
\hline Communication direction & Downlink & \\
\hline Central carrier frequency & $1.9 \mathrm{GHz}$ & {$[11]$} \\
\hline System bandwidth & $5 \mathrm{MHz}(N=25 \mathrm{PRBs})$ & [13] \\
\hline Noise power & $-112.4 \mathrm{dBm}$ & \\
\hline Link adaptation & LTE (15 MCSs) & {$[15],[16]$} \\
\hline Required cell-edge SNR & $-6.2 \mathrm{~dB}$ & [16] \\
\hline CSI knowledge & Perfect & \\
\hline $\mathrm{PF}$ time window $\left(\Delta_{\mathrm{PF}}\right)$ & 10 & \\
\hline Power time window $\left(\Delta_{\mathrm{AVG}}\right)$ & 10 & \\
\hline Traffic model & Full buffer & {$[11]$} \\
\hline Effective TTI duration & $1 \mathrm{~ms}$ & \\
\hline Snapshot duration & $1 \mathrm{~s}$ & \\
\hline Monte Carlo realizations & 150 & \\
\hline
\end{tabular}

Fig. 7 presents the total system spectral efficiency achieved by JOAS heuristic in comparison to traditional RRM schemes for several UEs per cell. As it is shown, the JOAS heuristic is able to overcome the improvement of performance achieved by both GRP and GRP+MS schemes for any UE density. As an example, with 16 UEs per cell, the total throughput gain is about $9 \%$. For 4 UEs per cell, there is only one D2D pair into each cell. Hence, a spatially incompatible D2D pair might be forcibly assigned with the former cellular UE by the GRP scheme, and with cellular UEs by the assignment step of the JOAS heuristic. The GRP+MS scheme has the flexibility of avoiding these harmful situations. However, it was not able to provide total performance improvements. In its turn, the improvement achieved by JOAS heuristic is due to the prioritization of cellular UEs which are in better conditions for sharing resources by the scheduling step. The benefits of resource sharing are promising in terms of the total system spectral efficiency, especially for high UE densities. But, there is a trade-off between the gains of enabling D2D communications and the impact on cellular communications.

Table IV presents the number of occurrences (in terms of percentage) of the D2D communications and the Jain's index

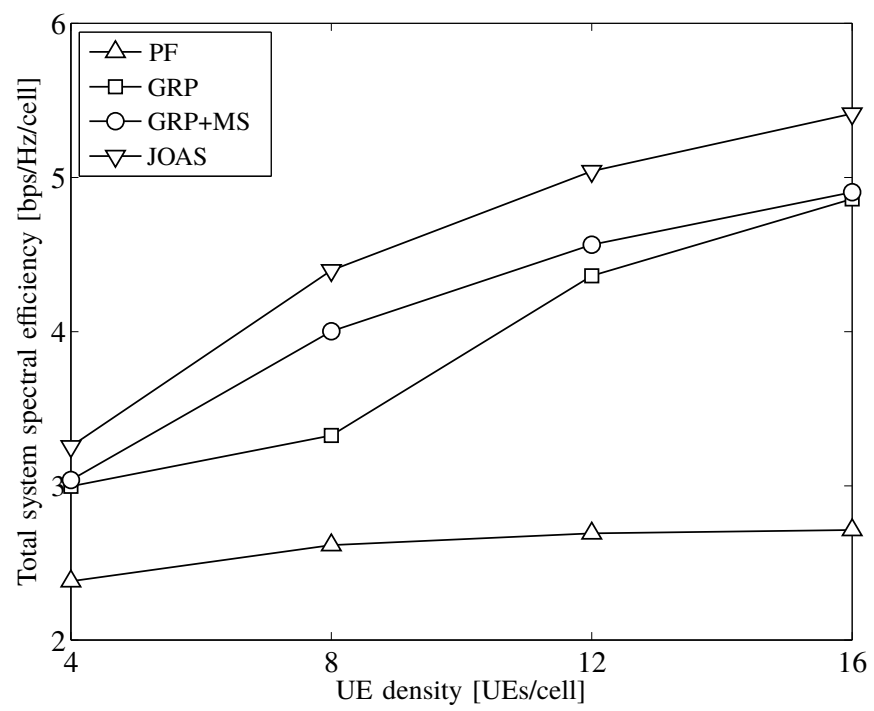

Fig. 7. Total system spectral efficiency of RRM schemes employing the ES assignment metric. The PF scheduling provides the performance in the conventional scenario without D2D communications underlaying the cellular network, while all other schemes have D2D communications enabled, such that, for example, considering $J=16 \mathrm{UEs} /$ cell, there exist $M=4 \mathrm{D} 2 \mathrm{D}$ pairs within each cell, because the percentage of D2D UEs is $50 \%$.

of fairness for cellular communications, which is defined as $\Pi=\left(\sum_{j}^{J} x_{j}\right)^{2} /\left(J \sum_{j}^{J} x_{j}^{2}\right)$, and $x_{j}=R_{j, 0, c, n}^{\mathrm{CELL}}$ [22]. The higher number of D2D communications achieved by GRP scheme greatly impacts on the fairness of cellular communications (compared to the PF in conventional scenario), which happens due to strong intra-cell interference induced by mode 2 . As mentioned in Section I, D2D links should not be mandatorily assigned in every radio resource, which is the case of GRP. In this context, GRP+MS scheme improves the performance of cellular communications by reducing the occurrences of D2D communications when it is not possible to achieve any gain in the total throughput. In its turn, the JOAS heuristic increases the occurrences of D2D communications and keeps the fairness of cellular communications, in comparison to the GRP+MS scheme. These results indicate the potential benefits brought by JOAS heuristic.

TABLE IV

NuMber OF OCCURRENCES OF D2D COMMUNICATIONS AND JAIN'S INDEX OF FAIRNESS FOR CELLULAR COMMUNICATIONS (\%)

\begin{tabular}{ccccccccc}
\hline & \multicolumn{4}{c}{ D2D occurrences } & \multicolumn{3}{c}{ Cellular fairness } \\
\cline { 2 - 8 } UEs/cell & PF & GRP & GRP+MS & JOAS & PF & GRP & GRP+MS & JOAS \\
\hline 8 & $\boldsymbol{x}$ & 100 & 53 & 71 & 45 & 12 & 31 & 31 \\
16 & $\boldsymbol{x}$ & 100 & 65 & 87 & 48 & 15 & 30 & 31 \\
\hline
\end{tabular}

Fig. 8 presents the performance of cellular and D2D communications achieved by JOAS heuristic and traditional RRM schemes. The GRP and GRP+MS schemes present almost the same total spectral efficiency. However, the impact on cellular communications is clearly higher with the GRP scheme because it does not consider the cellular throughput information. On the other hand, such impact is reduced with the GRP+MS scheme, so that the D2D throughput is reduced and the cellular 
one is improved. Moreover, the JOAS heuristic allows a higher impact on the throughput of cellular communications than the GRP+MS scheme to achieve a higher total throughput gain (recall that this is the main objetive of JOS problem), while maintaining the fairness of cellular communications, as it can be seen in Table IV.

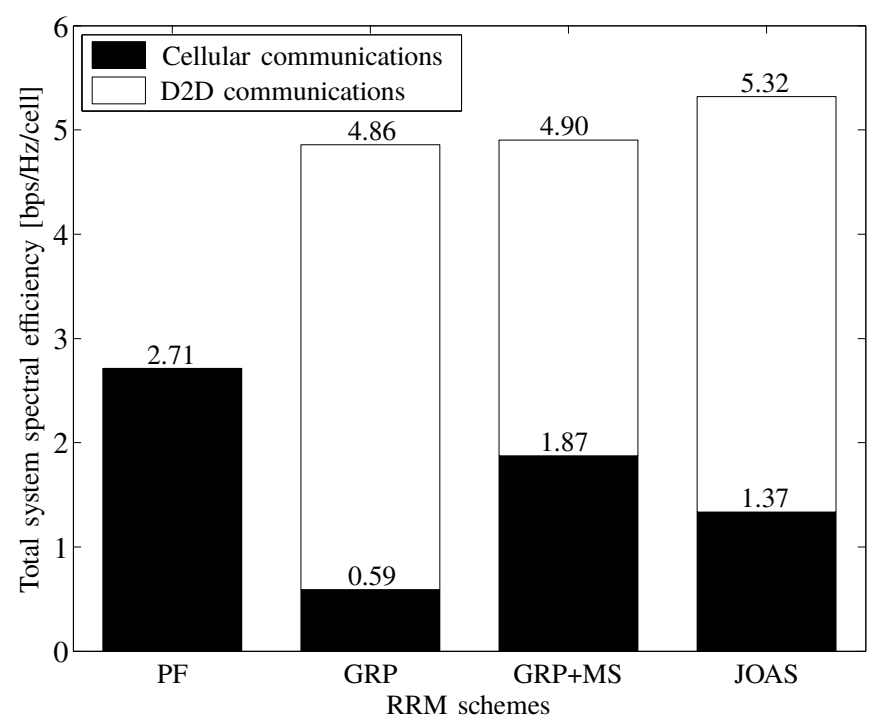

Fig. 8. Total system spectral efficiency of cellular and D2D communications for different RRM schemes using ES as assignment metric for $16 \mathrm{UEs} /$ cell.

Fig. 9 illustrates the total system spectral efficiency gains considering different RRM schemes and assignment metrics. When D2D communications occur in hotspot zones near the cell-edge is the most favorable scenario for resource sharing. Even though, the GRP scheme is not effective when using an assignment metric which deficiently measures the spatial compatibility among UEs, as the RND metric. Indeed, all the reliability of GRP remains on the efficiency of the assignment metric (see that the gap between GRP and GRP+MS schemes reduces for the ES case). On other hand, the scheduling step of JOAS heuristic is able to avoid the scheduling of D2D pairs inefficiently assigned by the RND assignment metric, which would highly harm the total throughput, giving the highest gap between JOAS and traditional RRM schemes, among the three assignment metrics. In summary, while JOAS heuristic improves in $10 \%$ the performance with GRP+MS scheme for the ES metric, the improvement is $26 \%$ for the RND metric.

Additionally, it is worth to notice that the simpler SLNR metric provides similar results as the more computationally demanding ES metric, which makes the former preferable to be implemented in real networks.

In order to diminish the processing complexity of the JOAS heuristic, pre-selection schemes are used to reduce the number of D2D pairs and groups evaluated by the assignment and scheduling steps, respectively.

Fig. 10 shows the total system spectral efficiency of the preselection scheme by varying the pre-assignment factor $F_{A}$ for the three considered assignment metrics. Inspecting the figure, even when a reduced number of cellular UEs is processed by the JOAS heuristic, e.g., $F_{A}=50 \%$ of the total (i.e., only 8 among 16 UEs in the cell), there is no performance loss

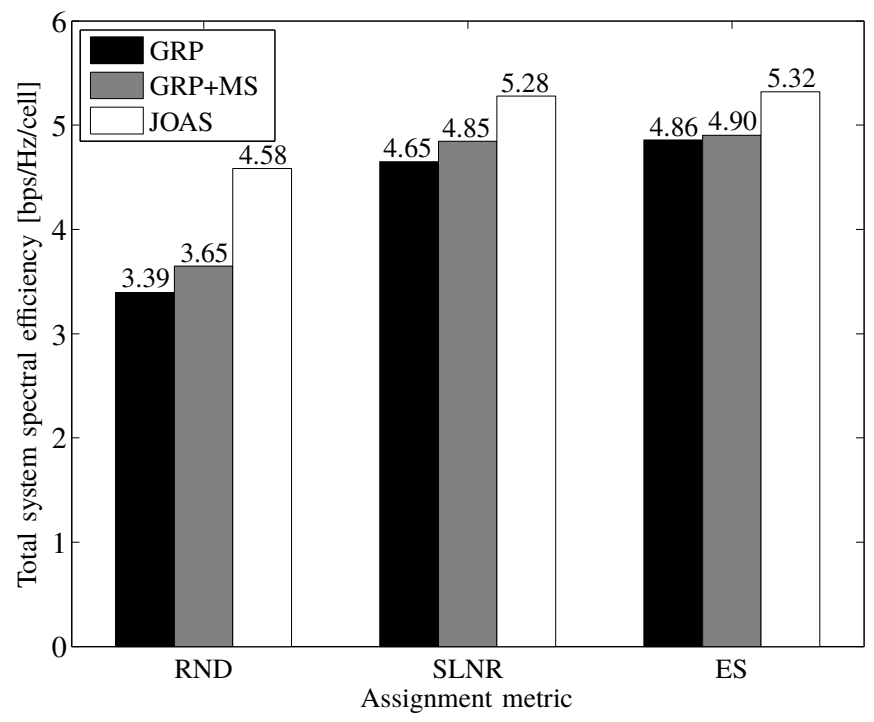

Fig. 9. Comparison between different assignment metrics for different RRM schemes, considering $16 \mathrm{UEs} / \mathrm{cell}$.

(in comparison to $F_{A}=100 \%$ ) for all assignment metrics. It means that the standard PF priority in cellular mode is efficient in pre-selecting the most likely UEs to be scheduled.

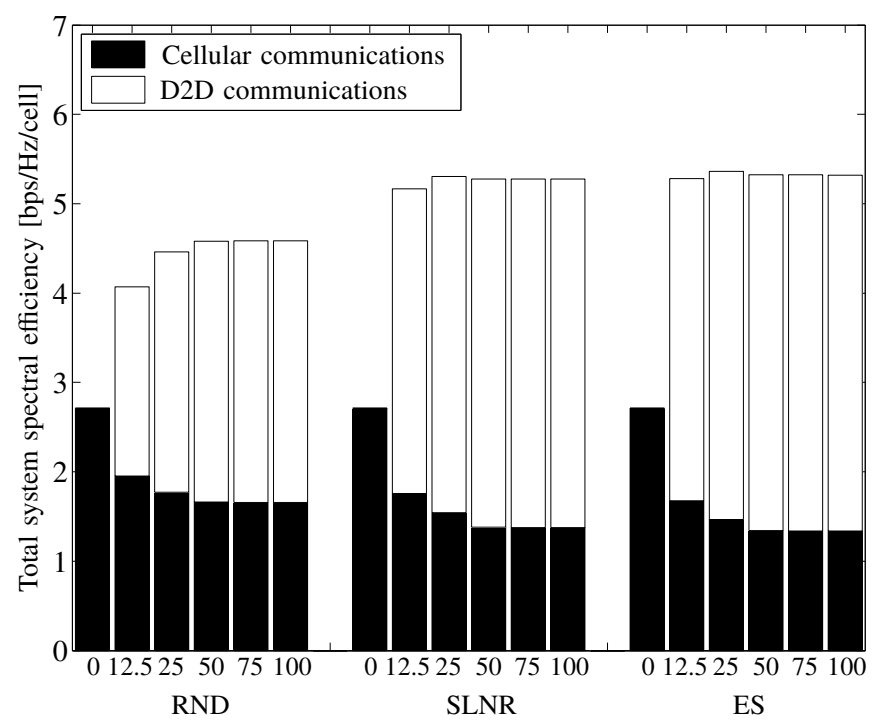

Fig. 10. Total system spectral efficiency of the pre-selection scheme by varying the pre-assignment factor $F_{A}[\%]$ and the assignment metric for JOAS heuristic, considering $16 \mathrm{UEs} / \mathrm{cell}$.

Furthermore, for $F_{A}=25 \%$ and SLNR metric, the preassignment scheme is reducing the probability of occurrences of D2D communications with some improvement for cellular communications while the total performance is almost kept constant. However, inefficient assignment metrics such as the RND metric tend to be more sensitive to pre-selection for very low pre-assignment factor values.

Fig. 11 presents the total system spectral efficiency of the pre-selection scheme by varying the pre-scheduling factor $F_{S}$ for the three considered assignment metrics. In the case of ES metric, the reduction in the amount of processed groups does not impact on the overall performance because it captures very 
well the group with the maximum priority in mode 2 . On the other hand, as the RND metric is already limited because it does not take into account any spatial compatibility on the assignment step, its total performance is further reduced when pre-scheduling limits the number of evaluated UEs. Finally, the SLNR metric, which does not fully capture all spatial compatibility as the ES metric, presents a considerable impact on the total performance, but only for pre-scheduling factor values lower than $F_{S}=50 \%$.

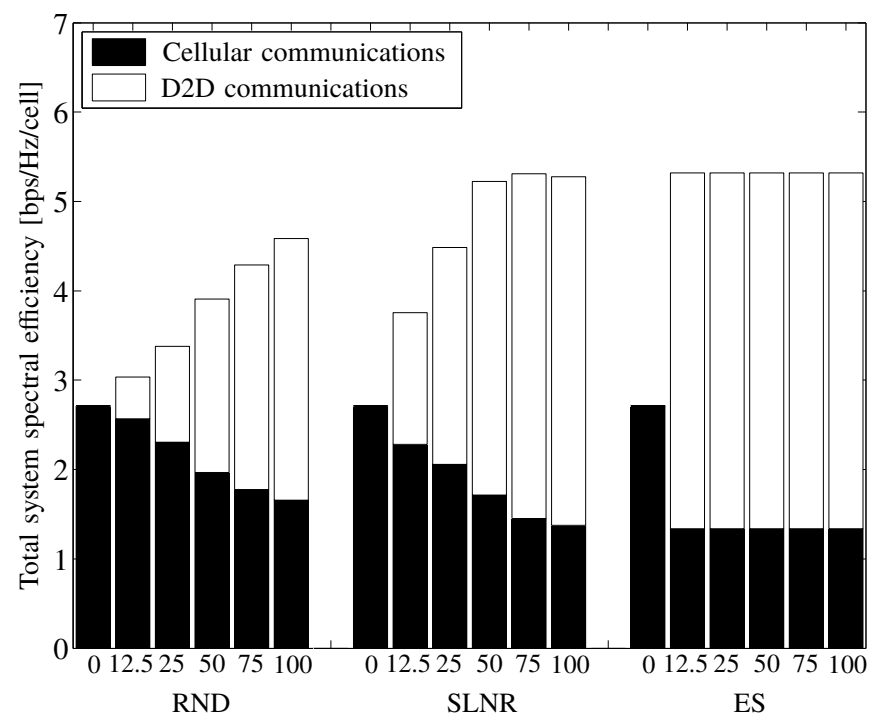

Fig. 11. Total system spectral efficiency of the pre-selection scheme by varying the pre-scheduling factor $F_{S}[\%]$ and the assignment metric for JOAS heuristic, considering $16 \mathrm{UEs} / \mathrm{cell}$.

As observed in all results, D2D links always impact on the performance of cellular communications even in favorable conditions. In the following, the protection mechanism for cellular communications, which is described in Section IV-D, to prevent excessive intra-cell interference from D2D communications, is evaluated. Fig. 12 provides the system spectral efficiency gains for cellular and D2D communications for different admissible impact factor values $F_{I}$.

Analyzing the figure, it may be seen that high total throughput gains are obtained with a minimum impact on the performance of cellular communications, as for $F_{I}=5 \%$. Even when a group achieves low values of system spectral efficiency and the impact on the estimated throughput of the cellular UE is small, the scheduling of the assigned D2D pair is avoided. Therefore, only D2D pairs in very good conditions for sharing resources can be scheduled.

Table $\mathrm{V}$ shows the occurrences of D2D communications, cellular throughput losses, and total throughput gains for low and high impact factor values, for 16 UEs per cell. As it can be seen, for $F_{I}=20 \%$, the JOAS heuristic presents the same number of D2D occurrences as the GRP+MS algorithm, but with lower cellular throughput loss and higher total throughput gain, which demonstrates its effectiveness.

\section{Conclusions}

The two main objectives of this work may be summarized as: 1) Propose a unified optimization problem for the JOS of

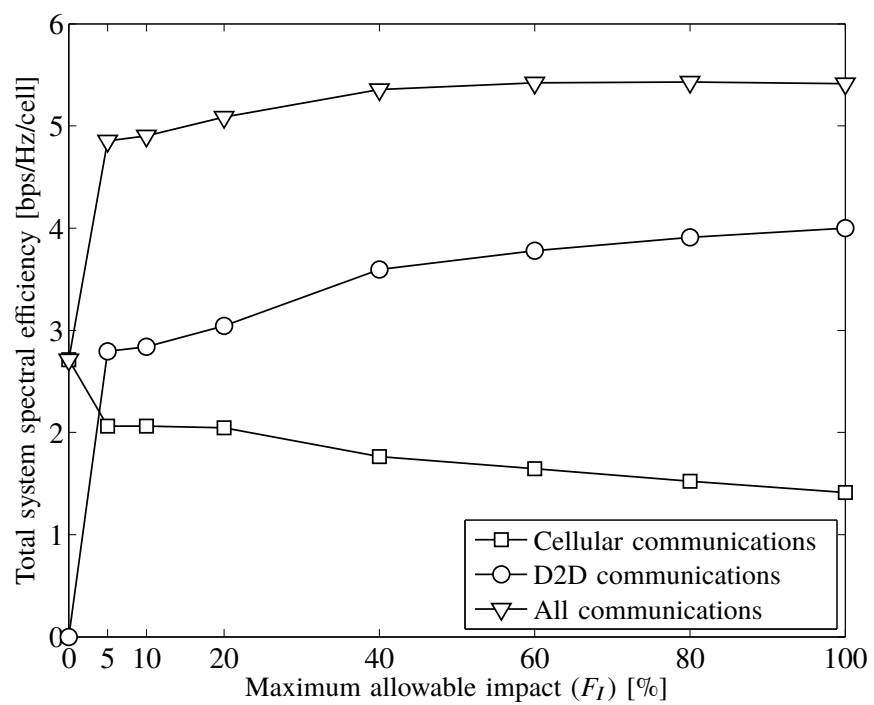

Fig. 12. Analysis of the protection mechanism for cellular communications by varying the impact factor $F_{I}[\%]$ and using the assignment metric ES for the JOAS heuristic, considering $16 \mathrm{UEs} / \mathrm{cell} . F_{I}=0 \%$ represents the baseline scenario, i.e., without any D2D communication.

TABLE V

Performance of the GRP+MS SCHEME AND PROTECTION MECHANISM OF JOAS HEURISTIC BY VARYING THE IMPACT FACTOR (\%).

\begin{tabular}{lcccc}
\hline & & \multicolumn{3}{c}{ JOAS } \\
\cline { 3 - 5 } & GRP+MS & $F_{\boldsymbol{I}}=5 \%$ & $F_{\boldsymbol{I}}=20 \%$ & $\boldsymbol{F}_{\boldsymbol{I}}=100 \%$ \\
\hline D2D occurrences & 65 & 59 & 65 & 87 \\
Cellular throughput loss $^{\mathrm{a}}$ & 31 & 24 & 25 & 48 \\
Total throughput gain $^{\mathrm{a}}$ & 81 & 79 & 88 & 100 \\
\hline
\end{tabular}

${ }^{a}$ Losses and gains are measured in comparison to the baseline scenario, as in Fig. 12.

cellular and D2D communications; 2) Elaborate a suboptimal JOAS heuristic for the JOS problem.

The JOS problem is a NP-hard combinatorial problem and, therefore, intractable, which makes low-complexity solutions necessary. In this way, JOAS heuristic was designed to jointly and opportunistically schedule both cellular and D2D communications in the same radio resource exploiting the spatial compatibility among UEs. Results showed that fairness, spectrum reuse, and the overall system capacity were improved with JOAS heuristic in comparison to traditional RRM schemes.

While the reliability of GRP scheme is intrinsically related with the efficiency of the assignment metric and mode selection (GRP+MS scheme) may still avoid the scheduling of a group in bad spatial conditions for sharing resources, the JOAS heuristic is less dependent of such metric. For example, the JOAS heuristic improved in $10 \%$ the performance obtained with GRP+MS scheme when using a good assignment metric, but the gain was about $26 \%$ for an unreliable metric. Indeed, the good performance of JOAS heuristic is more related with exploitation of the spatial organization of UEs, so that cellular UEs which are in very good sharing resource situations are opportunistically prioritized.

Furthermore, the JOAS heuristic achieved a considerable 
complexity reduction with minimum impact on the total throughput by pre-selecting UEs and/or groups that are most likely to be scheduled.

Also, to control the trade-off between the performance of cellular and D2D communications, it was possible to limit the number of occurrences of D2D links, so that they only happened when there were good conditions for sharing resources, i.e., the impact on cellular communications is minimum, but with high overall performance.

As future perspectives, the JOS problem may be investigated in more challenging scenarios: when multiple D2D communications of the same or from different hotspots are allowed per PRB into the same cell or using relays (i.e., more than onehop) [23], multiple layers are required to be processed and the scheduling problem tends to be more complex. Hence, the cooperation among cells of the same site such as in Coordinated Multipoint (CoMP) systems may be considered to jointly schedule multiple D2D pairs and the cellular UE.

\section{AcKNOWLeDGMENT}

This work is supported by the Innovation Center, Ericsson Telecomunicações S.A., Brazil, under EDB/UFC.33 and EDB/ UFC.40 Technical Cooperation Contracts. Also, Carlos Silva and José Mairton would like to acknowledge Coordenação de Aperfeiçoamento de Pessoal de Nível Superior (CAPES) for the financial support. Likewise, Tarcisio Maciel would like to acknowledge Conselho Nacional de Desenvolvimento Científico e Tecnológico (CNPq) for its financial support under the grants 426385/2016-0 and 308398/2015-7.

\section{REFERENCES}

[1] G. Fodor, E. Dahlman, G. Mildh, S. Parkvall, N. Reider, G. Miklós, and Z. Turányi, "Design aspects of network assisted device-to-device communications," IEEE Communications Magazine, vol. 50, no. 3, pp. 170176, Mar. 2012, DOI: https://doi.org/10.1109/MCOM.2012.6163598.

[2] J. M. B. da Silva, Jr., G. Fodor, and T. F. Maciel, "Performance analysis of network-assisted two-hop D2D communications," in IEEE Global Communications Conference Workshops (GC'14 Wkshps), Dec. 2014, pp. 1050-1056, DOI: https://doi.org/10.1109/GLOCOMW.2014.7063572.

[3] 3GPP, "Feasibility study for proximity services (ProSe)," 3rd Generation Partnership Project (3GPP), TR 22.803, Jun. 2013. [Online]. Available: http://www.3gpp.org/ftp/specs/html-info/22803.htm

[4] K. Doppler, M. Rinne, C. Wijting, C. B. Ribeiro, and K. Hugl, "Deviceto-device communication as an underlay to LTE-advanced," IEEE Communications Magazine, vol. 47, no. 12, pp. 42-49, Dec. 2009, DOI: https://doi.org/10.1109/MCOM.2009.5350367.

[5] M. Belleschi, G. Fodor, and A. Abrardo, "Performance analysis of a distributed resource allocation scheme for D2D communications," in IEEE Global Communications Conference Workshops (GC'11 Wkshps), Dec. 2011, pp. 358-362, DOI https://doi.org/10.1109/GLOCOMW.2011.6162471.

[6] R. L. Batista, C. F. M. e Silva, J. M. B. da Silva, Jr., T. F. Maciel, and F. R. P. Cavalcanti, "Impact of device-to-device communications on cellular communications in a multi-cell scenario," in XXXI Telecommunications Brazilian Symposium (SBrT2013), Fortaleza, Brazil, Sep. 2013, DOI: https://doi.org/10.14209/sbrt.2013.241.

[7] M. Zulhasnine, C. Huang, and A. Srinivasan, "Efficient resource allocation for device-to-device communication underlaying LTE network," in Proceedings of the IEEE Wireless and Mobile Computing, Networking and Communications (WiMob), Oct. 2010, pp. 368-375, DOI: https://doi.org/10.1109/WIMOB.2010.5645039.

[8] F. Wang, L. Song, Z. Han, Q. Zhao, and X. Wang, "Joint scheduling and resource allocation for device-to-device underlay communication," in Proceedings of the IEEE Wireless Communications and Networking Conference (WCNC), 2013, pp. 134-139, DOI: https://doi.org/10.1109/WCNC.2013.6554552.
[9] J. M. B. da Silva, Jr., T. F. Maciel, R. L. Batista, C. F. M. e Silva, and F. R. P. Cavalcanti, "UE grouping and mode selection for D2D communications underlaying a multicellular wireless system," in IEEE WCNC 2014 - Workshop on Device-to-Device and Public Safety Communications (WCNC'14 - WDPC Workshop), Istambul, Turkey, Apr. 2014, pp. 230-235, DOI: https://doi.org/10.1109/WCNCW.2014.6934891.

[10] G. Yu, L. Xu, D. Feng, R. Yin, G. Y. Li, and Y. Jiang, "Joint mode selection and resource allocation for device-to-device communications," IEEE Transactions on Communications, vol. 62, no. 11, pp. 3814-3824, Nov. 2014, DOI: https://doi.org/10.1109/TCOMM.2014.2363092.

[11] 3GPP, "Spatial channel model for multiple input multiple output (MIMO) simulations," 3rd Generation Partnership Project (3GPP), TR 25.996, Dec. 2009. [Online]. Available: http://www.3gpp.org/ftp/specs/html-info/25996.htm

[12] R. L. Batista, C. F. M. e Silva, J. M. B. da Silva, Jr., T. F. Maciel, and F. R. P. Cavalcanti, "What happens with a proportional fair cellular scheduling when D2D communications underlay a cellular network?" in IEEE WCNC 2014 - Workshop on Device-to-Device and Public Safety Communications (WCNC'14 - WDPC Workshop), Istambul, Turkey, Apr. 2014, pp. 260-265.

[13] 3GPP, "Physical layer aspect for evolved universal terrestrial radio access (UTRA)," 3rd Generation Partnership Project (3GPP), TR 25.814, Sep. 2006. [Online]. Available: http://www.3gpp.org/ftp/specs/htmlinfo/25814.htm

[14] — , "Evolved universal terrestrial radio access (E-UTRA); further advancements for E-UTRA physical layer aspects," 3rd Generation Partnership Project (3GPP), TR 36.814, Mar. 2010. [Online]. Available: http://www.3gpp.org/ftp/Specs/html-info/36814.htm

[15] "Evolved universal terrestrial radio access (EUTRA); physical layer procedures," 3rd Generation Partnership Project (3GPP), TS 36.213, Mar. 2008. [Online]. Available: http://www.3gpp.org/ftp/specs/html-info/36213.htm

[16] J. C. Ikuno, M. Wrulich, and M. Rupp, "System level simulation of LTE networks," in IEEE 71st Vehicular Technology Conference (VTC 2010-Spring), May 2010, pp. 1-5, DOI: https://doi.org/10.1109/VETECS.2010.5494007.

[17] J. Kim, D. Kim, and Y. Han, "Proportional fair scheduling algorithm for SC-FDMA in LTE uplink," in IEEE Global Communications Conference (GLOBECOM), 2012, pp. 4816-4820, DOI https://doi.org/10.1109/GLOCOM.2012.6503881.

[18] R. L. Batista, C. F. M. e Silva, J. M. B. da Silva, Jr., T. F. Maciel, and F. R. P. Cavalcanti, "Power prediction prior to scheduling combined with equal power allocation for the OFDMA UL," in Proceedings of 20th European Wireless (EW'14), Barcelona, Spain, May 2014.

[19] P. Belotti, C. Kirches, S. Leyffer, J. Linderoth, J. Luedtke, and A. Mahajan, "Mixed-integer nonlinear optimization," Mathematics and Computer Science Division, Preprint ANL/MCS-P3060-1112, Nov. 2012. [Online]. Available: http://www.mcs.anl.gov/papers/P30601112.pdf

[20] S. Sandberg, R. L. Batista, and A. Simonsson, "Coordinated multipoint transmission and reception (CoMP) in a wireless telecommunications network," International Patent PCT/SE2012/051 355, Dec., 2012.

[21] K. Doppler, C. B. Ribeiro, and J. Kneckt, "Advances in D2D communications: Energy efficient service and device discovery radio,' in 2nd International Conference on Wireless Communication, Vehicular Technology, Information Theory and Aerospace \& Electronic Systems Technology (Wireless VITAE'11), Mar. 2011, pp. 1-6, DOI: https://doi.org/10.1109/WIRELESSVITAE.2011.5940857.

[22] R. K. Jain, D.-M. W. Chiu, and W. R. Hawe, "A quantitative measure of fairness and discrimination for resource allocation in shared computer systems," Digital Equipment Corporation, Tech. Rep. DEC-TR-301, Sep. 1984. [Online]. Available: http://www.cse.wustl.edu/ jain/papers/ftp/fairness.pdf

[23] B. Zhou, H. Hu, S.-Q. Huang, and H.-H. Chen, "Intracluster device-todevice relay algorithm with optimal resource utilization," IEEE Transactions on Vehicular Technology, vol. 62, no. 5, pp. 2315-2326, Jun. 2013, DOI: https://doi.org/10.1109/TVT.2012.2237557. 


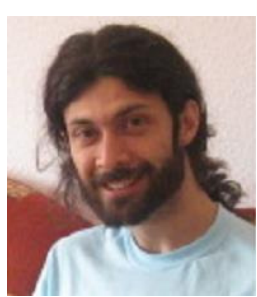

Rodrigo L. Batista received the B.Sc. degree in Computer Engineering from Federal University of Esprito Santo, Brazil, in 2008, and the M.Sc. degree in Teleinformatics Engineering from the Federal University of Ceará, Brazil, in 2011. We worked between 2009 and 2014 as a Research Engineer with the Wireless Telecom Research Group (GTEL), Brazil, in projects within technical cooperation with the Ericsson Research, Sweden. In 2012, he was a visiting researcher at Ericsson Research, Sweden, where he investigated the application of dynamic clustering techniques into cooperative systems. He has expertise on wireless networks simulation and related topics such as connection admission control and radio resource allocation for cooperative communications. His academic contributions include three patents, a book chapter, a journal paper, and conference papers.

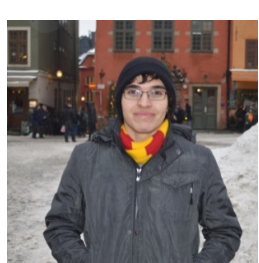

José Mairton B. da Silva Jr. received the B.Sc. (with honors) and M.Sc. degrees in Telecommunications Engineering from the Federal University of Ceará in Brazil (UFC) in 2012 and 2014, respectively. He worked as a researcher engineer at the Wireless Telecom Research Group (GTEL) in Fortaleza from 2012 to 2015, and during the $\mathrm{Au}-$ tumn/Winter of 2013-2014, he worked in an internship at Ericsson Research AB, Stockholm, on RRM procedures for device-to-device communications. He is currently a Ph.D. student at the Royal Institute of Technology (KTH) in Stockholm, Sweden. His research interests are on optimization for future cellular networks, including full-duplex communications, channel estimation, and massive MIMO.

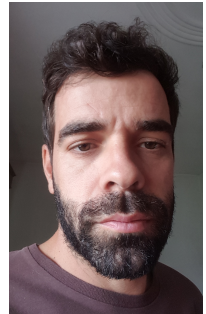

Carlos F. M. e Silva received a five years diploma and M.Sc. degrees in Electronics and Telecommunications Engineering from the University of Aveiro (UA), Portugal, in 2005 and 2010, respectively. In 2015 he also received a Ph.D. degree in Teleinformatics Engineering from the Federal University of Ceará (UFC), Brazil. Since 2006, Carlos Silva has been a researcher in several European projects, such as WINNER II (system requirements for beyond 3rd generation wireless networks), FUTON (RRM for wireless and optical networks), and COGEU (cognitive radio systems for efficient use and sharing of TVWS in the European context). Currently, he has a postdoc position at the Wireless Telecom Research Group (GTEL), Brazil, where he manages GTEL's team in the European-Brazilian project FUTEBOL and also works in cooperation projects with Ericsson Research. His main research interests include: spectrum usage optimization, scarcity and management, namely related with TV White Spaces (TVWS), Licensed Shared Access (LSA), Internet of Things (IoT) and Device-to-Device (D2D) communications; testbed experimentation; and architectural aspects of the future $5 \mathrm{G}$ networks.

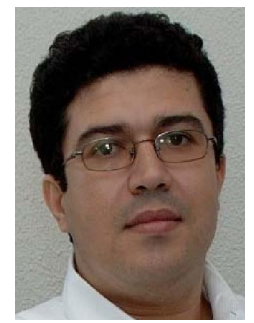

Tarcisio F. Maciel received his B.Sc. and M.Sc degrees in Electrical Engineering from the Federal University of Ceará (UFC) in 2002 and 2004, respectively, and his Dr.-Ing. degree from the Technische Universität Darmstadt (TUD), Germany, in 2008, also in Electrical Engineering. Since 2001, he has actively participated in several projects in technical and scientific cooperation between Wireless Telecom Research Group (GTEL), UFC, and Ericsson Research. From 2005 to 2008, he was a research assistant with the Communications Engineering Laboratory, TUD. Since 2008, he has been a member of the PostGraduation Program in Teleinformatics Engineering, UFC. In 2009, he was a Professor of computer engineering with UFC-Sobral and since 2010, he has been a Professor with the Center of Technology, UFC. His research interests include radio resource management, numerical optimization, and multiuser/multiantenna communications.

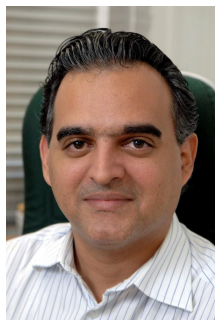

Francisco R. P. Cavalcanti received the B.Sc. and M.Sc. degrees in Electrical Engineering from Federal University of Ceará (UFC), Fortaleza, Brazil, in 1994 and 1996, respectively, and the D.Sc. degree in Electrical Engineering from the State University of Campinas, São Paulo, Brazil, in 1999. Upon graduation, he joined the UFC, where he is currently an Associate Professor and holds the Wireless Communications Chair with the Department of Teleinformatics Engineering. In 2000, he founded and, since then has directed the Wireless Telecom Research Group (GTEL), which is a research laboratory based on Fortaleza, which focuses on the advancement of wireless telecommunications technologies. At GTEL, he manages a program of research projects in wireless communications sponsored by the Ericsson Innovation Center in Brazil and Ericsson Research in Sweden. Prof. Cavalcanti has produced a varied body of work including two edited books, conference and journal papers, international patents and computer software dealing with subjects such as radio resource allocation, cross-layer algorithms, quality of service provisioning, radio transceiver architectures, signal processing and project management. Prof. Cavalcanti is a distinguished researcher of the Brazilian Scientific and Technological Development Council for his technology development and innovation record He also holds a Leadership and Management professional certificate from the Massachusetts Institute of Technology, Cambridge. 